ANNALES

POLONICI MATHEMATICI

$87(2005)$

\title{
Explicit bounds for the Kojasiewicz exponent in the gradient inequality for polynomials
}

\author{
by Didier D'Acunto (Pisa) and \\ KRZYSZTOF KuRdYKA (Le Bourget-du-Lac)
}

\begin{abstract}
Let $f: \mathbb{R}^{n} \rightarrow \mathbb{R}$ be a polynomial function of degree $d$ with $f(0)=0$ and $\nabla f(0)=0$. Eojasiewicz's gradient inequality states that there exist $C>0$ and $\varrho \in(0,1)$ such that $|\nabla f| \geq C|f|^{\varrho}$ in a neighbourhood of the origin. We prove that the smallest such exponent $\varrho$ is not greater than $1-R(n, d)^{-1}$ with $R(n, d)=$ $d(3 d-3)^{n-1}$.
\end{abstract}

1. Introduction. Let $f$ be an analytic function in a neighbourhood of the origin in $\mathbb{R}^{n}$ and assume $f(0)=0$ and $\nabla f(0)=0$. The well known Eojasiewicz gradient inequality (cf. [Ło1] or [Ło2]) states that there exist an open neighbourhood $U$ of the origin and two constants $C>0$ and $\varrho<1$ such that for any $x \in U$ we have

$$
|\nabla f(x)| \geq C|f(x)|^{\varrho} .
$$

The Eojasiewicz exponent of $f$ at the origin, denoted by $\varrho_{f}$, is the infimum of the exponents $\varrho$ satisfying the Łojasiewicz gradient inequality. Bochnak and Risler (cf. [Bo-Ri]) proved that $\varrho_{f}$ is a rational number. Moreover, inequality (1.1) holds with exponent $\varrho_{f}$ and some constant $C>0$. Knowing explicitly the exponent $\varrho_{f}$ is important for the study of the gradient flow near a singular point (cf. [Łol] and [KMP]).

We now assume that $f$ is a polynomial of degree $d$ in $n$ variables. It is known that $\varrho_{f}$ can be bounded by some rational number $\varrho(n, d)<1$ depending only on $n$ and $d$. If $f$ has an isolated zero at the origin (that is, $f$

2000 Mathematics Subject Classification: Primary 32Bxx, 34Cxx, Secondary 32Sxx, $14 \mathrm{P} 10$.

Key words and phrases: polynomials, Łojasiewicz's inequality, Łojasiewicz's exponent, valley lines. 00271

Supported by the EU research network IHP-RAAG contract number HPRN-CT-2001- 
has a strict local extremum at 0) J. Gwoździewicz [Gw] proved that

$$
\varrho_{f} \leq 1-\frac{1}{(d-1)^{n}+1} .
$$

In the present paper we consider the general case, that is, $f$ may have a non-isolated singularity at the origin. More precisely, for any integer $d \geq 2$ and for any polynomial $f$ in $n$ variables with $\operatorname{deg} f=d$ and $f(0)=0$ we have

Main TheOrem. The Eojasiewicz exponent $\varrho_{f}$ satisfies

$$
\varrho_{f} \leq 1-\frac{1}{d(3 d-3)^{n-1}} \text {. }
$$

More precisely $\varrho_{f} \leq 1-R(n, d)^{-1}$, where

$$
R(n, d)=\max \left\{d(3 d-4)^{n-1}, 2 d(3 d-3)^{n-2}\right\} .
$$

Our approach, unlike [Gw] which uses polar curves, is based on the study of ridge and valley lines attached to the singularity. More precisely, in a fixed non-critical level hypersurface $f^{-1}(t)$, we detect the points where the restriction of the function $|\nabla f|$ to $f^{-1}(t)$ has a local minimum. We denote by $\Gamma(f)$ the collection of all those points when $t$ varies in $\mathbb{R}$. As proved in [D'A-Ku] the set $\Gamma(f)$ is of dimension 1 for a generic polynomial of degree $d$. The set $\Gamma(f)$ is contained in the set of critical points of $|\nabla f|$ restricted to $f^{-1}(t)$, which is the set of points where $\nabla f$ is an eigenvector of $H_{f}$, the Hessian matrix of $f$.

The paper is organised as follows: first we explain the notion of ridge and valley lines and highlight some important properties of this set. Clearly the Łojasiewicz exponent $\varrho_{f}$ is reached on $\Gamma(f)$. Next we recall an elementary definition of multiplicity of intersection between a complex hypersurface $\{f=c\}$ and a complex algebraic curve containing $\Gamma(f)$; we prefer to call it the geometric degree of $f$ on $\Gamma(f)$. We state an important result on the semicontinuity of the geometric degree. We give a sketch of proof based on the existence of a stratification (in the complex algebraic case) satisfying Thom's $\left(a_{f}\right)$ condition.

We then compute the Łojasiewicz exponent first for a generic polynomial and finally, using semicontinuity of the geometric degree, for polynomials of a fixed degree on an algebraic family of curves of ridge and valleys.

After the submission of this paper A. Gabrielov informed us that he obtained the same bound in his paper [Ga]. (In fact he studied a more general case of pffafian sets). He used relative polar curves of the complexification of $f$ and $|\nabla f|^{2}$. It seems, however, that our proof is a bit more detailed and complete. For instance in the proof of Theorem 3.2 of $[\mathrm{Ga}]$ it is assumed that the minima of $f$ on the levels of $g$ (here $g=|\nabla f|^{2}$ ) intersected with a closed ball lie on the polar curve, but in fact they may occur on the boundary of 
the ball, so they may not belong to the polar curve. Even more generally it may happen that the relative polar curve may be empty; then one cannot apply the crucial Theorem 3.1 of [Ga].

It is important to stress that the difficulty is to find a bound valid for all polynomials (of fixed degree), since for a generic polynomial (which is Morse in fact) the exponent is equal to $1 / 2$.

The main difference between the proof in [Ga] and ours is the reduction of the dimension of $\Gamma(f)$. Since Gabrielov works with the complexification of $\Gamma(f)$, he can reduce to the case where $\operatorname{dim} \Gamma(f)=1$ by taking the intersection with a suitable complex 2-plane. Thus the curve on which the Łojasiewicz exponent is computed is not real in general, so it is not selected in $\Gamma(f)$. In our approach we actually obtain a real semialgebraic curve in $\Gamma(f)$ on which the order of $f$ can be controlled. This may be useful in further studies.

The problem of finding the exact maximal Łojasiewicz exponent $\varrho(n, d)$ for polynomials of degree $d$ in $n$ variables seems to be quite delicate. If we write a bound in the form $\varrho(n, d) \leq 1-R(n, d)^{-1}$ with $R(n, d)$ polynomial with respect to $d$, then as proved in the present paper $R(n, d)$ is of degree $n$. It seems interesting to estimate the leading coefficient. Probably it should be less than 1, as Gwoździewicz's [Gw] result suggests. However, the polynomials realizing $\varrho(n, d)$ may be extremely rare. Possibly there are finitely many of them (up to some natural equivalence relation).

Of course we do not claim that the bound we propose is optimal, but we hope that the method of valley lines (and their limits) may give a more precise estimate for the Łojasiewicz exponent.

Also lower bounds for $\varrho(n, d)$ seem to be unknown. One could conjecture that $\varrho(n, d) \geq 1-Q(n, d)^{-1}$ with $Q(n, d)$ polynomial of degree $n$ with respect to $d$. If $n=2$, then $Q(2, d)$ is of degree 2 and the leading coefficient of $Q(2, d)$ is not less than 15/28, by S. Gusein-Zade and N. Nekhoroshov [GuNe]. In this case the problem is related to $A_{N}$ singularities (with $N=N(d)$ maximal) which may appear on plane curves of degree $d$. Clearly for an $A_{N}$ singularity the Łojasiewicz exponent is $1-1 /(N+1)$.

2. Generalised valley lines. Let $f: \mathbb{R}^{n} \rightarrow \mathbb{R}$ be a polynomial with $\operatorname{deg} f=d \geq 2$. In a given level hypersurface of $f$ there are points of particular interest, namely the ridge and valley lines of $f$. A naive but important way of describing them is the following: fix a non-critical level hypersurface $f^{-1}(t)$ and consider the points $x \in f^{-1}(t)$ such that for all $s$ sufficiently close to $t$ the Euclidean distance $\operatorname{dist}\left(x, f^{-1}(s)\right)$ is greater than or equal to $\operatorname{dist}\left(x^{\prime}, f^{-1}(s)\right)$ for all $x^{\prime} \in f^{-1}(t)$ sufficiently close to $x$. We now give a more rigorous definition of the ridge and valley lines of $f$. 
Definition 2.1. We say that a point $x \in \mathbb{R}^{n}$ belongs to the ridge and valley set of $f$, denoted by $\Gamma_{1}(f)$, if the function $|\nabla f|^{2}$ restricted to $f^{-1}(f(x))$ has a local minimum at $x$.

This terminology is motivated by its analogy with elements of the Earth landscape.

Clearly, the ridge and valley set of $f$ is contained in the set

$$
\Theta_{1}(f)=\left\{x \in \mathbb{R}^{n}: d\left(|\nabla f|^{2}\right) \wedge d f=0\right\}
$$

of critical points of the function $|\nabla f|^{2}$ restricted to the level sets of $f$. Observe that $x \in \Theta_{1}(f)$ if and only $\nabla f(x)$ is an eigenvector of $H_{f}(x)$, the Hessian matrix of $f$ at $x$. Note that $\Theta_{1}(f)$ is a real algebraic set while the ridge and valley set is semialgebraic. The set $\Theta_{1}(f)$ is the set of common zeros of at most $n-1$ coefficients of the differential form $\omega=d\left(|\nabla f|^{2}\right) \wedge d f$.

Let $B^{n}\left(r_{0}\right)$ be the open ball of radius $r_{0}$ centred at the origin. Then the infimum of the function $|\nabla f|^{2}$ restricted to the hypersurfaces $f^{-1}(t) \cap B^{n}\left(r_{0}\right)$ is not necessarily reached inside $B^{n}\left(r_{0}\right)$ but maybe on the sphere $S^{n}\left(r_{0}\right)=$ $\partial B^{n}\left(r_{0}\right)$. This can occur when the origin is not an isolated singularity of $f$. One has to take into account some boundary effects. We therefore introduce the boundary ridge and valley set of $f$, denoted by $\Gamma_{2}(f)$, as the set of points at which the function $|\nabla f|^{2}$ restricted to $f^{-1}(t) \cap S^{n}\left(r_{0}\right)$ has a local minimum. Clearly, $\Gamma_{2}(f)$ is contained in

$$
\Theta_{2}(f)=\left\{x \in S^{n}\left(r_{0}\right): d\left(|\nabla f|^{2}\right) \wedge d f \wedge d r=0\right\},
$$

where $r(x)=|x|^{2}-r_{0}^{2}$. Then we define the generalised ridge and valley set of $f$ associated to $B^{n}\left(r_{0}\right)$ as $\Gamma(f)=\Gamma_{1}(f) \cup \Gamma_{2}(f)$. Clearly

$$
\Gamma(f) \subset \Theta(f)=\Theta_{1}(f) \cup \Theta_{2}(f) .
$$

The dimension of $\Theta(f)$ is not always equal to 1 . Nevertheless for a "generic" polynomial the set $\Theta(f)$ is an algebraic curve. Let $\mathbb{R}_{d}[\mathbf{X}]$ be the set of polynomials in $n$ variables of degree less than or equal to $d$ and $\mathbf{X}=\left(X_{1}, \ldots, X_{n}\right)$. Then we have

Proposition 2.2 ([D'A-Ku]). Fix integers $d, n \geq 2$. There is a semialgebraic set $G_{d} \subset \mathbb{R}_{d}[\mathbf{X}]$, with codim $G_{d} \geq 1$, such that for any polynomial $f \in \mathbb{R}_{d}[\mathbf{X}] \backslash G_{d}$, the set $\Theta(f)$ is of dimension 1 .

The proof of this proposition is based on transversality arguments and a detailed study of the space of symmetric matrices (see [D'A-Ku]). We shall call a polynomial $f$ of degree $d$ generic if $f \in \mathbb{R}_{d}[\mathbf{X}] \backslash G_{d}$.

When $\operatorname{dim} \Theta_{1}(f)=1$, the curve $\Theta_{1}(f)$ is contained in the set of common zeros of $n-1$ independent polynomials. As mentioned before, a point $x$ belongs to $\Theta_{1}(f)$ if and only if there exists $\lambda \in \mathbb{R}$ such that $H_{f}(x) \nabla f(x)=$ $\lambda \nabla f(x)$. Thus the $n-1$ polynomials describing $\Theta_{1}(f)$ have degree at most $3 d-4$. In the same way $\Theta_{2}(f)$ is contained in the set of common zeros of 
$n-2$ polynomial equations of degree at most $3 d-3$ and one polynomial equation of degree 2.

We shall need the following corollary of Proposition 2.2.

Corollary 2.3. For any polynomial $f \in \mathbb{R}_{d}[\mathbf{X}]$ there exists a polynomial mapping $\varphi: \mathbb{R} \rightarrow \mathbb{R}_{d}[\mathbf{X}]$ such that $\varphi(0)=f$ and for all but finitely many $t \in \mathbb{R}$ the polynomial is generic, that is, $\varphi(t) \notin G_{d}$.

Proof. Let $Z_{d}$ be the Zariski closure of $G_{d}$. Since $\operatorname{dim} Z_{d}=\operatorname{dim} G_{d}<n$, $Z_{d}$ is a proper algebraic subset of $\mathbb{R}_{d}[\mathbf{X}]$. By the classical curve selection lemma [Ło1] there exists an analytic mapping $\widetilde{\varphi}:(-a, a) \rightarrow \mathbb{R}_{d}[\mathbf{X}]$ such that $\tilde{\varphi}(0)=f$ and $\widetilde{\varphi}(0, s) \not \subset Z_{d}$. As $\varphi$ we can take the truncation of $\widetilde{\varphi}$ to a sufficiently high order.

3. Multiplicity of intersection. To prove that the estimate of the Main Theorem for the Łojasiewicz exponent holds true for both generic and non-generic polynomials we will use some basic facts from elementary intersection theory. First we make precise what we shall mean by the geometric degree of a polynomial $f: \mathbb{C}^{n} \rightarrow \mathbb{C}$ on an algebraic curve $\Gamma \subset \mathbb{C}^{n}$. Assume that $f$ is non-constant on any irreducible component of $\Gamma$. Then it is well known that for all but finitely many $z \in \mathbb{C}$ the number of points of $\Gamma \cap f^{-1}(z)$ is constant. We call this number the geometric degree of $f$ on $\Gamma$ and we denote it by $m(\Gamma, f)$. The next lemma follows easily from Rouché's Theorem.

Lemma 3.1. Let $\Gamma \subset \mathbb{C}^{n}$ be an algebraic curve and let $f: \mathbb{C}^{n} \rightarrow \mathbb{C}$ be a polynomial. Assume that $f$ is non-constant on any irreducible component of $\Gamma$. Let $\gamma: D \rightarrow \Gamma \subset \mathbb{C}^{n}$ be an injective holomorphic function defined in an open disk $D \subset \mathbb{C}$. Then the order of $f \circ \gamma$ at any point $s \in D$ is not greater than $m(\Gamma, f)$.

We now explain a kind of semicontinuity of the geometric degree. Consider an algebraic family $C_{t}, t \in \mathbb{C}^{*}$, of algebraic curves in $\mathbb{C}^{n}$. That is, we assume that the set

$$
C=\left\{(x, t) \in \mathbb{C}^{n} \times \mathbb{C}^{*}: x \in C_{t}\right\}
$$

is algebraic in $\mathbb{C}^{n} \times \mathbb{C}^{*}$. Let $C_{0}$ be the limit as $t \rightarrow 0$ of this family, precisely

$$
C_{0} \times 0=\bar{C} \backslash C,
$$

where the closure is taken in the Zariski topology in $\mathbb{C}^{n} \times \mathbb{C}$. In fact, since $C$ is constructible in $\mathbb{C}^{n} \times \mathbb{C}$ the closures of $C$ in the strong and the Zariski topologies are the same. Hence $C_{0}$ is an algebraic set in $\mathbb{C}^{n}$ of dimension $1=\operatorname{dim} C-1$.

Now let $f_{t}: \mathbb{C}^{n} \rightarrow \mathbb{C}, t \in \mathbb{C}$, be a family of polynomials such that the coefficients of $f_{t}$ are polynomials in $t$. Assume that, for any $t \in \mathbb{C}^{*}, f_{t}$ is non-constant on any irreducible component of $C_{t}$. 
LEMma 3.2. Let $\Gamma_{0}$ be the union of all irreducible components of $C_{0}$ on which $f_{0}$ is non-constant. Then

$$
m\left(\Gamma_{0}, f_{0}\right) \leq m\left(C_{t}, f_{t}\right)
$$

for any $t \neq 0$ sufficiently close to $0 \in \mathbb{C}$.

Proof. This result is a particular case of the general intersection theory (see for instance Chapter 11 in [Fu]). However, we shall give below a sketch of a simple geometric argument based on stratification theory. There exists (see for instance [Ha-Lê] or [HMS]) a stratification of $\left(C_{0} \times 0, C\right)$ which satisfies the so-called Thom's $\left(a_{f}\right)$ condition. In our case it means that there exists a finite set $B \subset C_{0}$ such that:

- $C_{0} \backslash B$ is smooth,

- for any $x \in C_{0} \backslash B$ and any $\varepsilon>0$ there exist a neighbourhood $U$ of $x$ and $\delta>0$ such that if $y \in C_{t} \cap U$ and $0<|t|<\delta$, then $C_{t}$ is smooth at $y$ and the distance between the tangent spaces $T_{y}\left(C_{t}\right)$ and $T_{x}\left(C_{0}\right)$ is less than $\varepsilon$.

Now choose $z \in \mathbb{C}$ such that the hypersurface $\left\{f_{0}=z\right\}$ meets $\Gamma_{0}$ transversally at the points of $\Gamma_{0} \backslash B$. So by definition, $m\left(\Gamma_{0}, f_{0}\right)$ is equal to the cardinality of $\Gamma_{0} \cap\left\{f_{0}=z\right\}$. Fix a point $x \in C_{0} \cap\left\{f_{0}=z\right\}$ and its small neighbourhood $U$. By transversality, if $t$ is close to 0 and $z^{\prime}$ is close to $z$, then $\left\{f_{t}=z^{\prime}\right\}$ and $C_{t}$ have at least one common point in $U$. This proves inequality (3.1).

4. Proof of the Main Theorem. In this section we use the generalised ridge and valley lines to bound the Łojasiewicz exponent $\varrho_{f}$ of a polynomial $f: \mathbb{R}^{n} \rightarrow \mathbb{R}$ of degree $d$.

Remark 4.1. Gwoździewicz $[\mathrm{Gw}]$ proved that if an analytic function $f$ has an isolated zero at the origin, then the Łojasiewicz exponent for the gradient inequality (1.1) is reached on all polar curves $P_{v}=(\nabla f)^{-1}(\mathbb{R} v)$ provided $v$ belongs to the complement in $\mathbb{R}^{n}$ of a proper linear subspace $L$. Moreover he gave examples showing that this is no longer true for non-isolated singularities. In particular he proved that if $f$ is a polynomial of degree $d$ with an isolated zero at the origin then $\varrho_{f}$ is bounded by $1-\left((d-1)^{n}+1\right)^{-1}$.

From now on we suppose that $f: \mathbb{R}^{n} \rightarrow \mathbb{R}$ is a polynomial of degree $d \geq 2$. We still assume that $f(0)=0$ and $\nabla f(0)=0$. Then we have

TheOREM 4.2. For any polynomial $f: \mathbb{R}^{n} \rightarrow \mathbb{R}$ of degree $d$ the Eojasiewicz exponent $\varrho_{f}$ at 0 is less than or equal to $1-\left(d(3 d-3)^{n-1}\right)^{-1}$. More precisely, if $f(0)=0$ and $\nabla f(0)=0$, then for any $r_{0}>0$ there exist $\varepsilon>0$ and $C>0$ such that

$$
|\nabla f(x)| \geq C|f(x)|^{\varrho}
$$

for any $x \in B^{n}\left(r_{0}\right)$ with $|f(x)|<\varepsilon$, where $\varrho=1-\left(d(3 d-3)^{n-1}\right)^{-1}$. 
The proof of Theorem 4.2 is based on the estimate of the order of contact of the hypersurface $f^{-1}(0)$ with a suitable parametrisation of the halfbranches of $\Gamma(f)$, the generalised ridge and valley set of $f$ in the ball $\bar{B}^{n}\left(r_{0}\right)$.

Precisely the following proposition is crucial.

Proposition 4.3. Let $f: \mathbb{R}^{n} \rightarrow \mathbb{R}$ be a polynomial of degree $d$. Assume that $(0, b) \subset f\left(B^{n}\left(r_{0}\right)\right)$ for some $b>0$. Then there exists an analytic arc $\theta:(-a, a) \rightarrow \mathbb{R}^{n}$ such that:

(1) $f(\theta(0))=0$ and $f \circ \theta((0, a)) \subset(0, b)$;

(2) if $s>0$ and $x \in f^{-1}(f(\theta(s))) \cap \bar{B}^{n}\left(r_{0}\right)$, then $|\nabla f(x)| \geq|\nabla f(\theta(s))|$;

(3) $\operatorname{ord}_{0}(f \circ \theta) \leq d(3 d-3)^{n-1}$.

Proof of Theorem 4.2. Assume we have proved Proposition 4.3 and let $\theta$ be an analytic curve as in the proposition.

We write $\theta(t)=a_{m} t^{m}+a_{m+1} t^{m+1}+\cdots$ with $\left|a_{m}\right|=1, m \geq 1$. For small $s>0$ we put

$$
\gamma(s)=\theta\left(s^{1 / m}\right)=a_{m} s+a_{m+1} s^{(m+1) / m}+\cdots,
$$

which is a convergent Puiseux series. Note that $\left|\gamma^{\prime}(s)\right| \rightarrow 1$ as $s \rightarrow 0$. Let us write the Puiseux expansion of $f \circ \gamma$ :

$$
(f \circ \gamma)(s)=\alpha_{\nu} s^{\nu}+\alpha_{k} s^{k}+\cdots,
$$

with $\nu \leq(1 / m) d(3 d-3)^{n-1}, k \in(1 / m) \mathbb{N}, k>\nu, \alpha_{\nu}>0$. Then we have $(f \circ \gamma)^{\prime}(s)=\left\langle\nabla f(\gamma(s)), \gamma^{\prime}(s)\right\rangle$ and $\left|\gamma^{\prime}(s)\right| \simeq 1$ as $s \rightarrow 0$. This implies

$$
|\nabla f(\gamma(s))| \geq c \nu \alpha_{\nu} s^{\nu-1} \simeq f(\gamma(s))^{(\nu-1) / \nu}
$$

for some positive constant $c>0$ and small $s>0$. So there exists $C>0$ such that

$$
|\nabla f(\gamma(s))| \geq C|f(\gamma(s))|^{(\nu-1) / \nu} .
$$

Recall that if $x \in f^{-1}(f(\gamma(s)))$, then $|\nabla f(x)| \geq|\nabla f(\gamma(s))|$. Thus we have

$$
|\nabla f(x)| \geq C|f(x)|^{(\nu-1) / \nu}
$$

for any $x \in B^{n}\left(r_{0}\right)$ such that $f(x)>0$ is small enough. Replacing $f$ by $-f$ we obtain the result also for $f(x)<0$.

REMARK 4.4. Note that, for a fixed $\operatorname{ord}_{0}(f \circ \theta)$, the exponent $\varrho=$ $(\nu-1) / \nu$ is the largest possible when $m=1$, that is, the curve $\Gamma(f)$ is smooth.

To complete the proof of Theorem 4.2 it remains to prove Proposition 4.3. We shall distinguish two cases. Namely, we first consider the case of a generic polynomial and then, using the results of Section 3, we extend our bound on the Lojasiewicz exponent to the non-generic polynomials.

Proof of Proposition 4.3. We will use the notations and results from Section 2. Clearly the arc $\theta$ must be chosen from the algebraic set $\Theta(f)$. 
CASE 1: The polynomial $f$ is generic. That is, $\Theta(f)$ is actually a curve. We have

LEMMA 4.5. Assume that $f$ is generic, and let $\theta:(-a, a) \rightarrow \Theta(f) \subset \mathbb{R}^{n}$ be an analytic arc. Suppose that the complexification of $\theta$ is injective in a small disk around the origin in $\mathbb{C}$. Then $\operatorname{ord}_{0}(f \circ \theta) \leq d(3 d-3)^{n-1}$.

Recall that $\Theta(f)=\Theta_{1}(f) \cup \Theta_{2}(f)$ is the union of two algebraic sets. So the image of $\theta$ entirely lies in one of them.

First we assume that $\theta(s) \in \Theta_{1}(f)$ for all $s \in(-a, a)$. Recall that if $f$ is generic then $\Theta_{1}(f)$ is of dimension one and is contained in the common zeros of $n-1$ independent coefficients of the 2-form $\omega=d\left(|\nabla f|^{2}\right) \wedge d f$. Denote them by $g_{1}, \ldots, g_{n-1}$; recall that $\operatorname{deg} g_{i} \leq 3 d-4$. We may assume that $\nabla g_{1}(x), \ldots, \nabla g_{n-1}(x)$ are linearly independent for all but finitely many $x \in \Theta_{1}(f)$.

Indeed, pick a smooth point $x_{i}$ in each irreducible component $C_{i}$ of $\Theta_{1}(f)$. Let $g_{1}, \ldots, g_{n-1}$ be such that $\nabla g_{1}\left(x_{1}\right), \ldots, \nabla g_{n-1}\left(x_{1}\right)$ are linearly independent, and suppose that $\nabla g_{1}, \ldots, \nabla g_{n-1}$ are linearly dependent at $x_{2}$. Let $h_{1}, \ldots, h_{n-1}$ be coefficients of $\omega$ such that $\nabla h_{1}\left(x_{2}\right), \ldots, \nabla h_{n-1}\left(x_{2}\right)$ are linearly independent.

Now put $\widetilde{g}_{i}=g_{i}+\varepsilon h_{i}$. If $\varepsilon>0$ is small enough, then the gradients of $\widetilde{g}_{i}$ are linearly independent at both points $x_{1}, x_{2}$. Continuing in this way we obtain linear combinations $p_{1}, \ldots, p_{n-1}$ of the coefficients of $\omega$ such that their gradients are linearly independent at each point $x_{i}$, hence, by algebraicity, at all but finitely many points of $\Theta_{1}(f)$.

From Bézout's Theorem (cf. e.g. [Be-Ri], [Fu]) we obtain

$$
m\left(\Theta_{1}(f), f\right) \leq \operatorname{deg} f \prod_{i=1}^{n-1} \operatorname{deg} g_{i} \leq d(3 d-4)^{n-1} \leq d(3 d-3)^{n-1} .
$$

Hence by Lemma 3.1,

$$
\operatorname{ord}_{0}(f \circ \theta) \leq m\left(\Theta_{1}(f), f\right) \leq d(3 d-3)^{n-1} .
$$

Assume now $\theta(s) \in \Theta_{2}(f)$ for all $s \in(-a, a)$. Recall that $\Theta_{2}(f)$ is contained in the common zeros of $n-2$ polynomial equations of degree at most $3 d-3$ and one polynomial equation of degree 2. So again by Bézout's Theorem,

$$
m\left(\Theta_{2}(f), f\right) \leq 2 d(3 d-3)^{n-2} \leq d(3 d-3)^{n-1}
$$

and by Lemma 3.1,

$$
\operatorname{ord}_{0}(f \circ \theta) \leq 2 d(3 d-3)^{n-2} \leq d(3 d-3)^{n-1} .
$$

This proves Lemma 4.5.

We now continue the proof of Proposition 4.3 in the first case. Choose $\varepsilon>\operatorname{such}$ that $(0, \varepsilon) \subset f\left(B^{n}\left(r_{0}\right)\right)$. By the classical curve selection lemma (cf. 
e.g. [BCR] $)$ there exists a semialgebraic curve $\tilde{\theta}:(0, \varepsilon) \rightarrow \bar{B}^{n}\left(r_{0}\right)$ such that

$$
|\nabla f(x)| \geq|\nabla f(\widetilde{\theta}(s))|, \quad x \in f^{-1}(\widetilde{\theta}(s)) \cap \bar{B}^{n}\left(r_{0}\right) .
$$

It follows from Puiseux's Theorem (cf. e.g. [Ło3]) that there exists a rational number $q>0$ such that $\theta(s)=\widetilde{\theta}\left(s^{q}\right), s>0$, has an analytic (holomorphic) extension which is actually injective on a small disk around the origin.

Observe that $\theta(s) \in \Gamma(f) \subset \Theta_{1}(f) \cup \Theta_{2}(f)$. But $\theta$ is analytic and $\Theta_{1}(f), \Theta_{2}(f)$ are algebraic sets, so either

$$
\theta(s) \in \Theta_{1}(f), s \in(0, \varepsilon), \quad \text { or } \quad \theta(s) \in \Theta_{2}(f), s \in(0, \varepsilon),
$$

for $\varepsilon>0$ small enough. From (4.2) and (4.4) we obtain

$$
\operatorname{ord}_{0}(f \circ \theta) \leq d(3 d-3)^{n-1} \text {. }
$$

This proves Proposition 4.3 in Case 1, that is, for a generic polynomial.

CAsE 2: The general case. We now consider an arbitrary polynomial $f: \mathbb{R}^{n} \rightarrow \mathbb{R}$, of degree $d \geq 2$. By Proposition 2.2 there exists an algebraic family of polynomials $f_{t}, t \in \mathbb{R}$, of degree at most $d$ such that $f_{0}=f$ and for all sufficiently small $t \neq 0$ the polynomial $f_{t}$ is generic in the sense of Section 2. Actually, by Corollary 2.3 we may choose this family so that the coefficients of $f_{t}$ are polynomials in $t$.

Hence $f_{t}$ is a well defined polynomial on $\mathbb{C}^{n}$ of degree at most $d$. So we have two associated algebraic families of curves $C_{t}=\Theta_{1}\left(f_{t}\right), t \in \mathbb{C}^{*}$, and $D_{t}=\Theta_{2}\left(f_{t}\right), t \in \mathbb{C}^{*}$.

More precisely, $C_{t}$ or $D_{t}$ is a curve for all but finitely many $t \in \mathbb{C}^{*}$. This follows from the fact that the genericity in Proposition 2.2 comes from a transversality condition which is valid also in the complex case.

Let $C_{0}$ and $D_{0}$ be the respective limits, as $t \rightarrow 0$, of the families $C_{t}=$ $\Theta_{1}\left(f_{t}\right), t \in \mathbb{C}^{*}$, and $D_{t}=\Theta_{2}\left(f_{t}\right), t \in \mathbb{C}^{*}$. Note that $C_{0}$ and $D_{0}$ are algebraic curves in $\mathbb{C}^{n}$. We set

$$
\Gamma_{0}(f)=\mathbb{R}^{n} \cap\left(C_{0} \cup D_{0}\right) .
$$

LEMMA 4.6. Let $y \in \mathbb{R}$ and assume that $f^{-1}(y) \cap \bar{B}^{n}\left(r_{0}\right) \neq \emptyset$. Then there exists a point $x_{0} \in \Gamma_{0}(f) \cap \bar{B}^{n}\left(r_{0}\right)$ such that

$$
|\nabla f(x)| \geq\left|\nabla f\left(x_{0}\right)\right|, \quad x \in f^{-1}(y) \cap \bar{B}^{n}\left(r_{0}\right) .
$$

To prove the lemma observe that $\Gamma_{0}(f) \cap \bar{B}^{n}\left(r_{0}\right)$ is the Hausdorff limit, as $t \rightarrow 0$, of the family $\left(C_{t} \cup D_{t}\right) \cap \bar{B}^{n}\left(r_{0}\right), t \in \mathbb{R}^{*}$. Recall that if $f_{t}^{-1}(y) \cap \bar{B}^{n}\left(r_{0}\right)$ is non-empty then $\left|\nabla f_{t}\right|$, restricted to $f_{t}^{-1}(y) \cap \bar{B}^{n}\left(r_{0}\right)$, has a minimum at a point which belongs to $\left(C_{t} \cup D_{t}\right) \cap \bar{B}^{n}\left(r_{0}\right)=\Theta\left(f_{t}\right) \cap \bar{B}^{n}\left(r_{0}\right)$. We leave the details to the reader.

We are now in a position to finish the proof of Proposition 4.3 in the second case. As in Case 1 we can choose in $\Gamma_{0}(f)$ an analytic arc $\theta(s)$, $s \in(-a, a)$, which satisfies condition (2) in Proposition 4.3. Clearly, as in 
Case 1, there are two possibilities: the image of $\theta$ is included in $C_{0}$ or in $D_{0}$. Recall that, by (4.1),

$$
m\left(\Theta_{1}\left(f_{t}\right), f_{t}\right) \leq d(3 d-4)^{n-1} \leq d(3 d-3)^{n-1}, \quad t \neq 0
$$

So, by the semicontinuity of intersection (Lemma 3.2), we have

$$
m\left(C_{0}, f\right) \leq d(3 d-4)^{n-1} \leq d(3 d-3)^{n-1}
$$

Hence if the image of $\theta$ is included in $C_{0}$ it follows from Lemma 3.1 that

$$
\operatorname{ord}_{0}(f \circ \theta) \leq m\left(C_{0}, f\right) \leq d(3 d-3)^{n-1} .
$$

Analogously by (4.3) we have

$$
m\left(\Theta_{2}\left(f_{t}\right), f_{t}\right) \leq 2 d(3 d-3)^{n-2} \leq d(3 d-3)^{n-1}, \quad t \neq 0 .
$$

Again, by Lemma 3.2, we have

$$
m\left(D_{0}, f\right) \leq 2 d(3 d-3)^{n-1} \leq d(3 d-3)^{n-1} .
$$

Hence if the image of $\theta$ is included in $D_{0}$ it follows from Lemma 3.1 that

$$
\operatorname{ord}_{0}(f \circ \theta) \leq m\left(D_{0}, f\right) \leq d(3 d-3)^{n-1} \text {. }
$$

This ends the proof of Proposition 4.3.

Note that $\operatorname{ord}_{0}(f \circ \theta)$ is actually bounded by $\max \left\{d(3 d-4)^{n-1}\right.$, $\left.2 d(3 d-3)^{n-2}\right\}$. So as claimed in the statement of the Main Theorem we have precisely $\varrho_{f} \leq 1-R(n, d)^{-1}$, where

$$
R(n, d)=\max \left\{d(3 d-4)^{n-1}, 2 d(3 d-3)^{n-2}\right\} .
$$

REMARK 4.7. Note that, in contrast to the geometric degree, the Eojasiewicz exponent is not upper semicontinuous. Consider for instance the family

$$
f_{t}(x)=t x^{2}+x^{d}
$$

Clearly $\varrho_{f_{t}}=1 / 2$ for $t \neq 0$ and $\varrho_{f_{0}}=1-1 / d$.

So in the proof of Theorem 4.2 we cannot claim that we first prove the estimate for a generic polynomial and then we extend it "by continuity" to all polynomials. Actually a generic polynomial is a Morse function, hence its Łojasiewicz exponent is equal to $1 / 2$. What we can control is the geometric degree of the polynomial on an algebraic curve on which the Eojasiewicz exponent is reached.

\section{References}

[Be-Ri] R. Benedetti and J.-J. Risler, Real Algebraic and Semialgebraic Sets, Act. Math., Hermann, Paris, 1990.

[BCR] J. Bochnak, M. Coste and M. F. Roy, Real Algebraic Geometry, Ergeb. Math. Grenzgeb. 36, Springer, 1998. 
[Bo-Ri] J. Bochnak et J.-J. Risler, Sur les exposants de Eojasiewicz, Comment. Math. Helv. 50 (1975), 493-507.

[D'A-Ku] D. D'Acunto and K. Kurdyka, Bounds for gradient trajectories of polynomial and definable functions with applications, preprint, Univ. de Savoie, 2004.

[Fu] W. Fulton, Intersection Theory, Springer, 1998.

[Ga] A. Gabrielov, Multiplicities of Pfaffian intersections, and the Eojasiewicz inequality, Selecta Math. (N.S.) 1 (1995), 113-127.

[GuNe] S. M. Gusein-Zade and N. N. Nekhoroshov, On singularities of type $A_{k}$ on simple curves of fixed degree, Funct. Anal. Appl. 34 (2000), 214-215.

[Gw] J. Gwoździewicz, The Eojasiewicz exponent of an analytic function at an isolated zero, Comment. Math. Helv. 74 (1999), 364-375.

[Ha-Lê] H. A. Hamm et D. T. Lê, Un théorème de Zariski du type de Lefschetz, Ann. Sci. École Norm. Sup. (4) 6 (1973), 317-355.

[HMS] J. P. G. Henry, M. Merle et C. Sabbah, Sur la condition de Thom stricte pour un morphisme analytique complexe, Ann. Sci. École Norm. Sup. 17 (1984), $227-268$.

[KMP] K. Kurdyka, T. Mostowski and A. Parusiński, Proof of the gradient conjecture of R. Thom, Ann. of Math. 152 (2000) 763-792.

[Ło1] S. Łojasiewicz, Ensembles semi-analytiques, preprint IHES, 1965.

[Ło2] - Sur les trajectoires du gradient d'une fonction analytique, in: Geometry Seminars, 1982-1983, Univ. Stud. Bologna, Bologna, 1984, 115-117.

[Ło3] - Introduction to Complex Analytic Geometry, Birkhäuser, Basel, 1991.

Dipartimento di Matematica

Università degli Studi di Pisa

Via Filippo Buonarroti, 2

56127 Pisa, Italy

E-mail: didier.dacunto@univ-savoie.fr
Laboratoire de Mathématiques LAMA UMR 5127 CNRS, Université de Savoie 73376 Le Bourget-du-Lac Cedex, France E-mail: krzysztof.kurdyka@univ-savoie.fr

Reçu par la Rédaction le 19.11.2004

Révisé le 27.11.2005 